\title{
Assessment of Coronary Flow Reserve During Angioplasty Using A Doppler Tip Balloon Catheter. Comparison With Digital Subtraction Cineangiography
}

\author{
PATRICK W. SERRUYS, M.D., FELIX ZIJLSTRA, M.D., HANS H. C. REIBER, M.D. \\ RENE KONING, M.D., and JOS ROELANDT, M.D.
}

From the Catheterization Laboratory and Laboratory for Clinical and Experimental Image Processing,

Thoraxcenter, Erasmus University, The Netherlands.

\begin{abstract}
Intracoronary blood flow velocity measurements with a Doppler probe and the radiographic assessment of myocardial perfusion with contrast media previously have been used to investigate regional coronary flow reserve. In the present study we applied both techniques in the same patients to measure the immediate improvement in coronary flow reserve as a result of angioplasty. In addition we compared papaverine induced hyperemia with reactive hyperemia following transient transluminal occlusion of a major coronary artery. In 13 consecutive patients with a single proximal stenosis, coronary flow reserve was measured pre- and postangioplasty by digital subtraction cineangiog. raphy, while pre-and postangioplasty Doppler measurements before and after papaverine were obtained in the proximal part of the stenotic vessel After the last transluminal occlusion, reactive hyperemia recorded with the Doppler probe was also compared to the coronary flow reserve measurement obtained during papaverine induced hyperemia. As a result of the angioplasty, coronary flow reserve measured with the radiographic technique
\end{abstract}

\section{Introduction}

Since the introduction of coronary angioplasty in $1977,{ }^{1}$ this procedure has gained increasing importance in the treatment of coronary artery obstructions. So far, the immediate results of this procedure have been assessed by coronary angiog-

Address for reprints: P.W. Serruys, M.D., Ph.D., Catheterization Laboratory and Laboratory for Clinical and Experimental Image Processing Thoraxcenter, Erasmus University, P.0. Box 1738, 3000 DR Rotterdam. The Netherlands.

Submitted for publication November 18, 1987; accepted January $25,1988$. (mean $\pm S D)$ increased from $1.1 \pm 0.4$ to $2.2 \pm 0.4$ $(P<0.001)$, while coronary flow reserve measured with the Doppler probe (mean $\pm S D$ ) increased from $1.2 \pm 0.3$ to $2.4 \pm 0.4(P<0.001)$. Pharmacologically induced hyperemia measured with the radiographic technique and the Doppler probe were linearly related $(r=0.91$ with a SEE 0.3) and confirmed the reliability of the intracoronary measurements. Using these two independent techniques, coronary flow reserve immediately after angioplasty was found to be substantially improved but still abnormal. In addition, the magnitude of hyperemia induced by papaverine was comparable to the reactive hyperemia following transluminal occlusion, although the latter measurement was recorded with the angioplasty catheter still across the dilated lesion. (J Interven Cardiol, 1988:1:1)

angioplasty, coronary flow reserve, digital subtraction cineangiography, Doppler tip balloon catheter

raphy and the residual pressure gradient. However, the change in luminal size of an artery following the mechanical disruption of its internal wall cannot be assessed accurately from the detected angiographic contours. ${ }^{2,3}$ Also, while the measured residual pressure gradient may have long-term prognostic value, it reflects only the hemodynamic state at rest. ${ }^{4,5}$ Recently the assessment of coronary flow reserve has been proposed as a better method to evaluate the functional consequences of a coronary artery obstruction. ${ }^{6-9}$

Papaverine currently is regarded as the ideal vasodilator for the induction of maximal hyperemia, since its intracoronary administration results in a 
potent and short-lasting hyperemia. ${ }^{10,11}$ Intracoronary blood flow velocity measurements with a Doppler probe and the radiographic assessment of myocardial perfusion with contrast media have previously been used to investigate regional coronary flow reserve. ${ }^{12-18}$ In the present study we applied both techniques to measure the immediate improvement in coronary flow reserve as a result of PTCA, and to compare the papaverine induced hyperemia with the reactive hyperemia induced by the transient transluminal occlusion of a major coronary artery.

\section{Patients and Methods}

Fourteen patients undergoing elective PTCA for angina pectoris were studied. All patients had evidence of myocardial ischemia as indicated either by ECG changes at rest or at exercise and/or during exercise thallium scintigraphy. Informed consent was obtained for the additional investigations. All patients were studied without premedication, but their medical treatment (nitrates, calcium antagonists, and beta blockers) was continued on the day of the procedure. No adequate intracoronary blood flow velocity could be recorded, despite recanalization and satisfactory dilatation, in one patient with a totally occluded left anterior descending coronary artery. This patient was excluded from further analyses.

\section{Protocol of the Investigational Procedure}

1. Coronary cineangiography was performed in at least two, preferably orthogonal, projections for quantitative analysis of the coronary artery stenosis.

2. Coronary flow reserve was assessed by digital subtraction cineangiography (DSC), following the induction of pharmacological hyperemia (PH) by $12.5 \mathrm{mg}$ papaverine injected through the guiding catheter $\left(8 \mathrm{~F}\right.$, Angiomedic $\left.{ }^{\mathrm{R}}\right)$.

3. A long guidewire (length: $315 \mathrm{~cm}$, diameter: 0.014 inch) was passed through the coronary artery stenosis.

4. A balloon catheter with a Doppler probe (DOP) at the tip was advanced over the guidewire into the coronary artery to measure coronary blood flow velocity. The precise location of the tip of the balloon catheter with respect to the stenotic lesion, immediately proximal to the lesion and beyond any major side branches, was determined by injection of a contrast medium. After recording the baseline intracoronary blood flow velocity, $\mathbf{P H}$ was induced by injecting $12.5 \mathrm{mg}$ papaverine through the guiding catheter. The ratio of peak mean intracoronary blood flow velocity to baseline (PH-DOP) was then determined.

5. Thereafter, the balloon was advanced across the stenosis and 3 to 6 inflations and up to 12 atmospheres were used to dilate the stenosis until repeat cineangiography showed a good result ( $<50 \%$ diameter stenosis). The mean total inflation time was $172 \mathrm{~s} /$ patient (range: 148-352s).

6. During and following each balloon inflation, coronary blood flow velocity was recorded continuously with the Doppler probe situated across the stenotic lesion. Reactive hyperemia immediately after the final balloon deflation $(\mathrm{RH})$ was calculated as the ratio of maximal mean intracoronary blood flow velocity to stabilized blood flow velocity, observed after the hyperemia had subsided.

7. With the Doppler tip pulled back into the proximal part of the coronary artery, PH-DOP was assessed again.

8. After removal of the balloon catheter and the guidewire, coronary flow reserve was measured with digital subtraction cineangiography (PHDSC, as in 2).

9. Coronary cineangiography was repeated postPTCA in the same projections as used at the start of the procedure (see 1) for quantitative analysis of the coronary artery stenosis.

\section{Quantitative Analysis of the Coronary Artery}

The determination of coronary arterial dimensions from $35 \mathrm{~mm}$ cinefilm was performed with a computer-based Cardiovascular Angiography Analysis System (CAAS), previously described in detail. ${ }^{16,19,20}$ In essence, boundaries of a selected coronary artery segment are detected automatically from optically magnified and video digitized regions of interest of a cineframe. The absolute diameter of the stenosis in millimeters is deter- 
mined using the guiding catheter as a scaling device. This involves the automatic edge-detection of the boundaries of the catheter in situ and the comparison of this value with the actual diameter measurement of the catheter using a micrometer. Calibration of the diameter in absolute values $(\mathrm{mm})$ is achieved by comparing the mean diameter of the guiding catheter in pixels with the measured size in millimeters. Each catheter is measured individually. ${ }^{21}$ To correct the detected contour of the arterial and catheter segments for pincushion distortion, a correction vector is computed for each pixel based on a computer-processed cineframe with a centimeter grid placed against the input screen of the image intensifier. ${ }^{20}$ Since the functional significance of a stenosis is related to the expected normal cross-sectional area of the vessel at the point of obstruction, we use a computer estimation of the original arterial dimension at the site of the obstruction to define the interpolated reference regions. ${ }^{19,20}$ The interpolated percentage area stenosis (AS) and the minimal luminal cross-sectional area $\left(\mathrm{mm}^{2}\right)$ are then calculated for at least two, preferably othogonal, projections. The length of the lesion is then determined form the diameter function on the basis of a curvature analysis. A mean of 2.3 angiographic projections/patients were used in this study.

Coronary Flow Reserve Measurements with Digital Subraction Cineangiography (CFR-DSC). The coronary flow reserve measurement from $35 \mathrm{~mm}$ cinefilm has been implemented on the CAAS. ${ }^{16}$ The heart was atrially paced at a rate just above the spontaneous heart rate. An ECG-triggered injection into the coronary artery was made with Iopamidol at $37^{\circ} \mathrm{C}$ through a Medrad Mark IV ${ }^{R}$ infusion pump. This nonionic contrast agent has a viscosity of $9.4 \mathrm{cP}$ at $37^{\circ}$, an osmolarity of 0.796 osm.kg, ${ }^{1}$ and an iodine content of $370 \mathrm{mg} / \mathrm{mL}$. For the left coronary artery, $7 \mathrm{~mL}$ of contrast was injected at a flow rate of $4 \mathrm{~mL} / \mathrm{s}$, and the coronary angiogram obtained in the left anterior oblique projection. For the right coronary artery, $5 \mathrm{~mL}$ was injected at a flow rate of $3 \mathrm{~mL} / \mathrm{s}$, and the angiogram taken in the left or right anterior oblique projection. The injection rate of the contrast medium was judged to be adequate if back flow of contrast medium into the aorta occurred. The angiogram was repeated $30 \mathrm{~s}$ after $\mathrm{PH}$, produced by a bolus injection of $12.15 \mathrm{mg}$ papaverine into the coronary artery by way of the guiding catheter. Five or six consecutive end-diastolic cineframes were selected for analysis. Logarithmic nonmagnified mask-mode background subtraction was applied to the image subset to eliminate noncontrast medium densities. The last end-diastolic frame prior to the administration of contrast was chosen as the mask. From the sequence of background subtracted images, a contrast arrival time image was determined using an empirically derived fixed density threshold. ${ }^{16}$ Each pixel was labeled with the sequenced number for the cardiac cycle numbered from the cycle of the ECG-triggered contrast injection in which the pixel intensity level first exceeded the threshold. In addition to the contrast arrival time image, a density image was computed, with each pixel intensity value being representative of the maximal local contrast medium accumulation. The CFR-DSC was defined as the ratio of the regional flow computed from a hyperemic image divided by the regional flow of the corresponding baseline image.

Regional flow of values were quantitatively determined using the following videodensitometric principle: regional block flow $(\mathrm{Q})=$ regional vascular volume/transit time. Regional vascular volume was assessed from logarithmic mask-mode subtraction images, using the Lamberg Beer relationship. CFR can then be calculated as:

$$
\mathrm{CFR}=\frac{\mathrm{Qh}}{\mathrm{Qb}}=\frac{\mathrm{CDh}}{\mathrm{ATh}}: \frac{\mathrm{CDb}}{\mathrm{ATb}}
$$

where $C D$ is the mean contrast density and AT the mean appearance time at baseline (b) and hyperemia (h). Mean contrast medium appearance time and density were computed within a user-defined region of interest, which was chosen so that the epicardial coronary arteries visible on the angiogram, the coronary sinus, and the great cardiac vein were all excluded from the analysis. ${ }^{16}$

\section{Intracoronary Blood Flow Velocity Measurements}

A $20 \mathrm{MHz}$ ultrasonic crystal mounted on the tip of the angioplasty catheter was used in all patients. Recently Sibley et al. ${ }^{22}$ validated clinically and experimentally the ability of a similar catheter with an end-mounted piezoelectric crystal to provide 


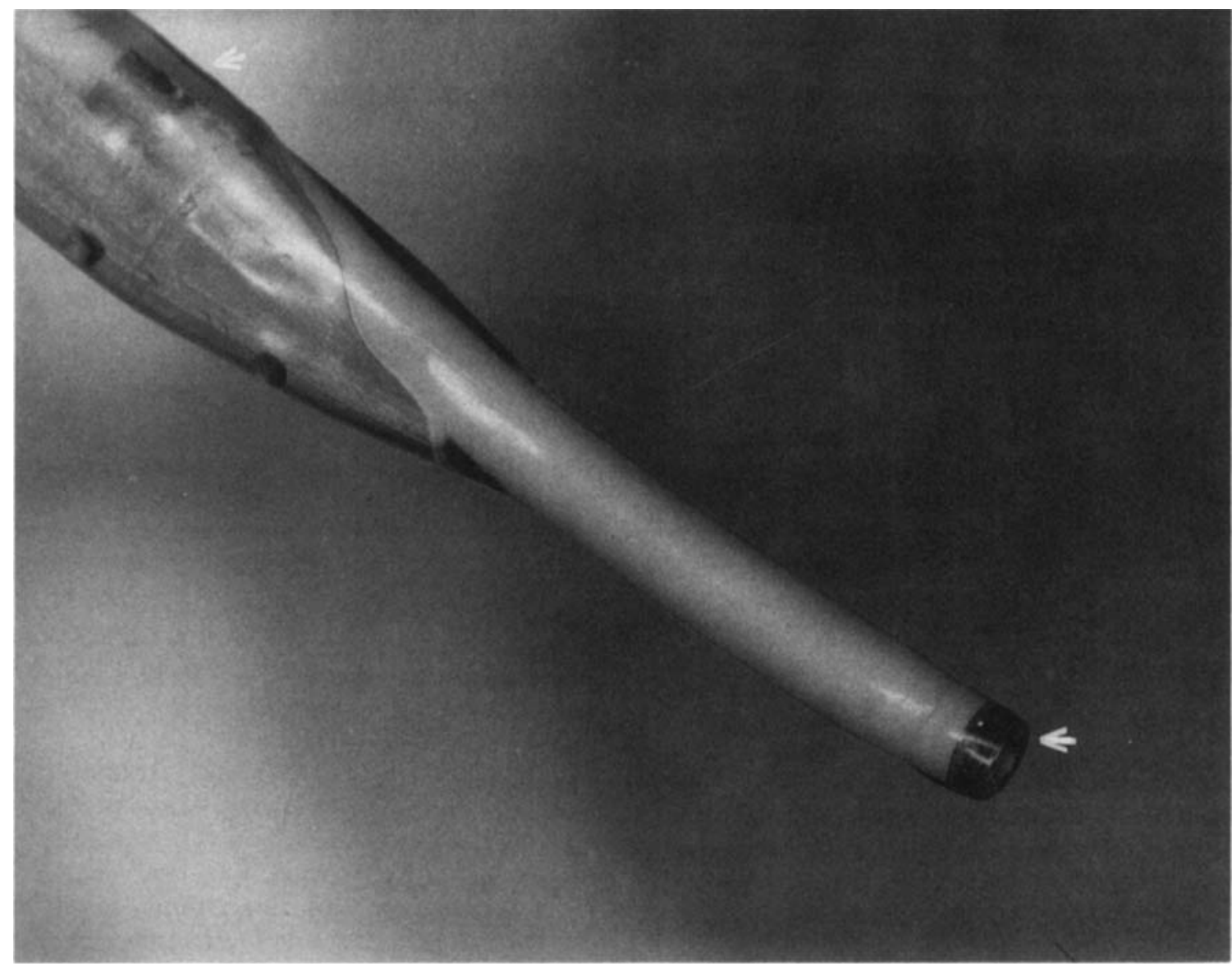

Figure 1A. Tip of a Doppler-balloon catheter showing the end-mounted ultrasonic crystal and a radiopaque marker within the balloon.

accurate continuous on-line measurement of coronary blood flow velocity and vasodilator reserve. ${ }^{22}$ A balloon diameter size of $3.0 \mathrm{~mm}$ was used in ten patients, and $3.4 \mathrm{~mm}$ in three patients. The crosssectional area of the catheter was measured by means of a micrometer at the tip, at the site of the balloon, and in the shaft of the catheter. The cross-sectional area at the three different sites were respectively: $1.2 \mathrm{~mm},{ }^{2} 0.65 \mathrm{~mm},{ }^{2}$ and $1.5 \mathrm{~mm}^{2}$. The Doppler crystal is a $1.0-\mathrm{mm}$ diameter annulus with a $0.5-\mathrm{mm}$ central hole (Fig. 1). Two leads are soldered to the crystal and pass through the catheter between the original $0.5-\mathrm{mm}$ lumen and a thin-walled tube which serves as a new $0.4-\mathrm{mm}$ lumen. The leads exit near the proximal luer hub and are wired to a two pin plug for connection to the pulsed Doppler instrument. The connector cable contains an integral torroidal isolation trans- former that insulates the patient from the instrument and provides impedance matching for more efficient energy transfer. The new inner lumen extends from the luer hub through the crystal providing a smooth unobstructed path for a guidewire. Blood flow velocity is measured from the catheter tip transducer using a range-gated 20 $\mathrm{MHz}$ pulsed Doppler designed specially for this purpose (Baylor College of Medicine). The master oscillator frequency of $20 \mathrm{MHz}$ is pulsed at a frequency of $62.5 \mathrm{KHz}$. Each pulse is approximately one $\mathrm{ms}$ in width and therefore contains 20 cycles of the master oscillator frequency. The parameter chosen (master oscillator frequency $=20 \mathrm{MHz}$ and pulse repetition frequency $=62.5 \mathrm{MHz}$ ) allows velocities up to $100 \mathrm{~cm} / \mathrm{s}$ to be recorded at a distance of up to $1 \mathrm{~cm}$ from the catheter tip. The sampling window was individually adjusted to ob- 


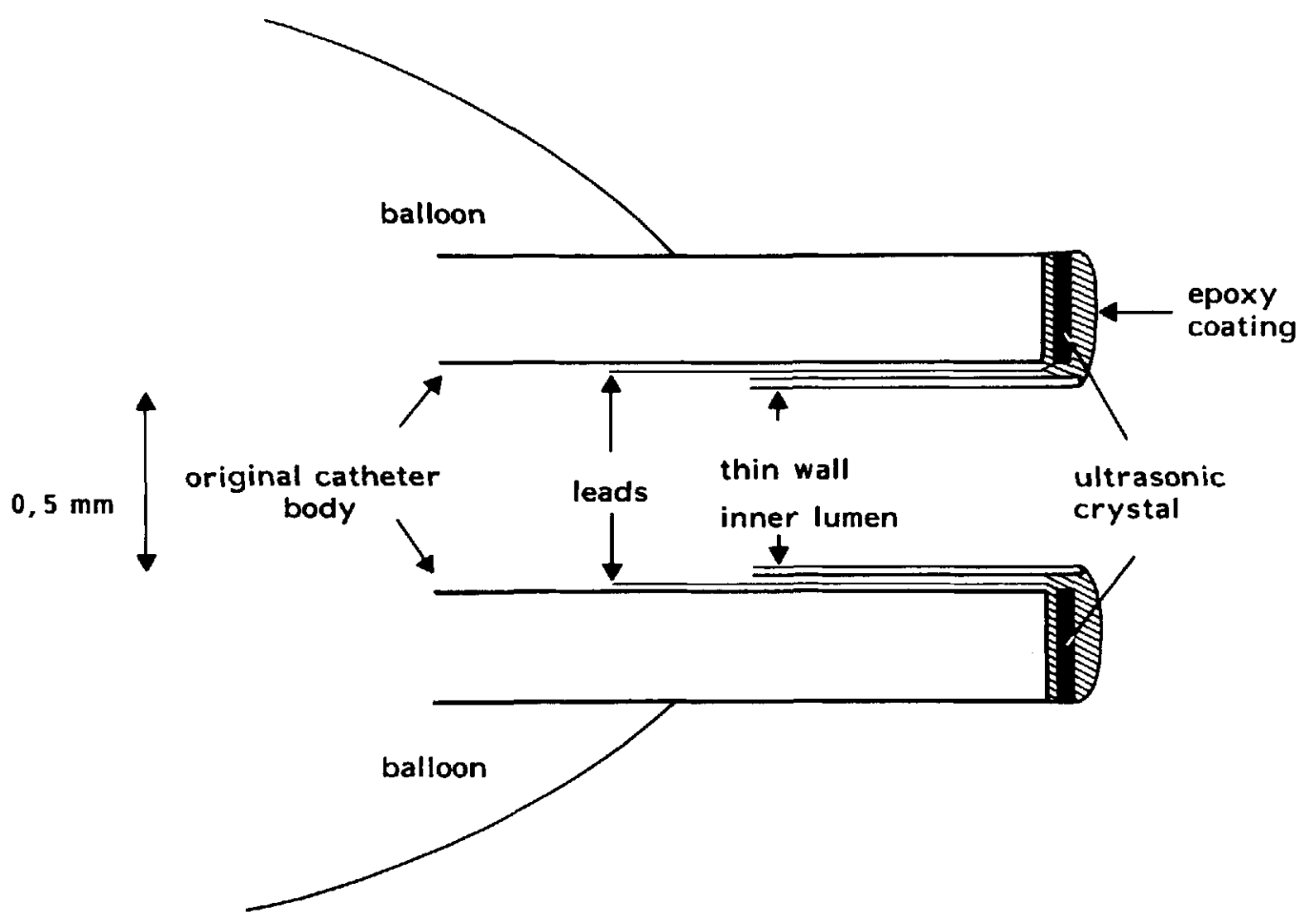

Figure 1B. Schematic cross-sectional drawing of Doppler-ballon catheter.

tain the most optimal signal that (usually) resulted in a sampling window of $1.18 \mathrm{~mm}$ (range 1.15-2.0). The output of the pulsed Doppler is a frequency shift $(\Delta \mathrm{f}, \mathrm{KHz})$ that can be related to blood velocity by the Doppler equation: $\Delta F R=2 F(V / c) \cos$ a, where $F$ is the uitrasonic frequency $(20 \mathrm{MHz}), \mathrm{V}$ is the velocity within the sample volume, $\mathrm{c}$ is the speed of sound in blood $(1.500 \mathrm{~m} / \mathrm{s})$, and $\mathrm{a}$ is the angle between the velocity vector and the sound beam. Using an end-mounted crystal with the catheter parallel $\left( \pm 20^{\circ}\right.$ to the vessel axis, cos $\mathrm{a}=1$ $\pm 6 \%$, and the relation between the Doppler shift and velocity is approximately $3.75 \mathrm{~cm} / \mathrm{s}$ per $\mathrm{kHz}^{22}$ (Fig. 2A). Previous calibration experiments in canine femoral and coronary arteries have shown that the measured Doppler shift frequency is proportional to volume flow measured by timed collection. $^{12,14,18,22}$

\section{Statistical Methods}

Comparisons between data were made with the Student's $t$-test for paired observation. Linear re- gression analyses were used to define the relationships between the various functional measurements.

\section{Results}

Clinical Data. The mean age of the 13 patients was 56 years (range: 43-69), 10 were male. Eleven patients had single-vessel coronary artery disease and two patients had two-vessel disease. The investigated and dilated coronary artery was the left anterior descending artery in nine patients, the circumflex artery and the right coronary artery in two patients each. The mean left ventricular ejection fraction was $67 \%$ and ranged from $38 \%-81 \%$. The length (mean $\pm \mathrm{SD}$ ) of the stenotic lesions was $8.10 \pm 1.9 \mathrm{~mm}$ and did not change as a result of angioplasty. In one patient the coronary arteriogram showed grade II/IV collateral filling of the angioplasty vessel and in two patients grade III/IV. ${ }^{23}$

In 12 of the 13 patients wall motion of the myocardial region supplied by the dilated vessel was 


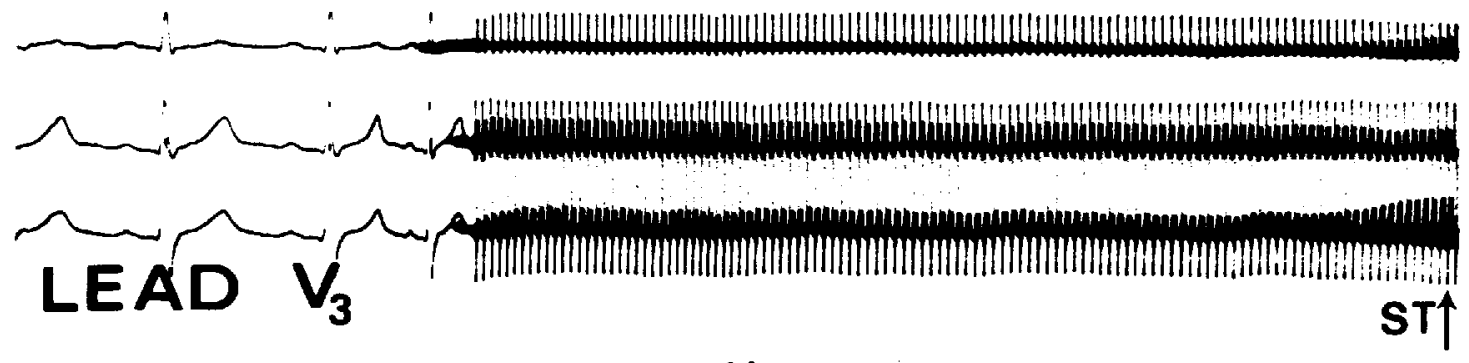

MEAN
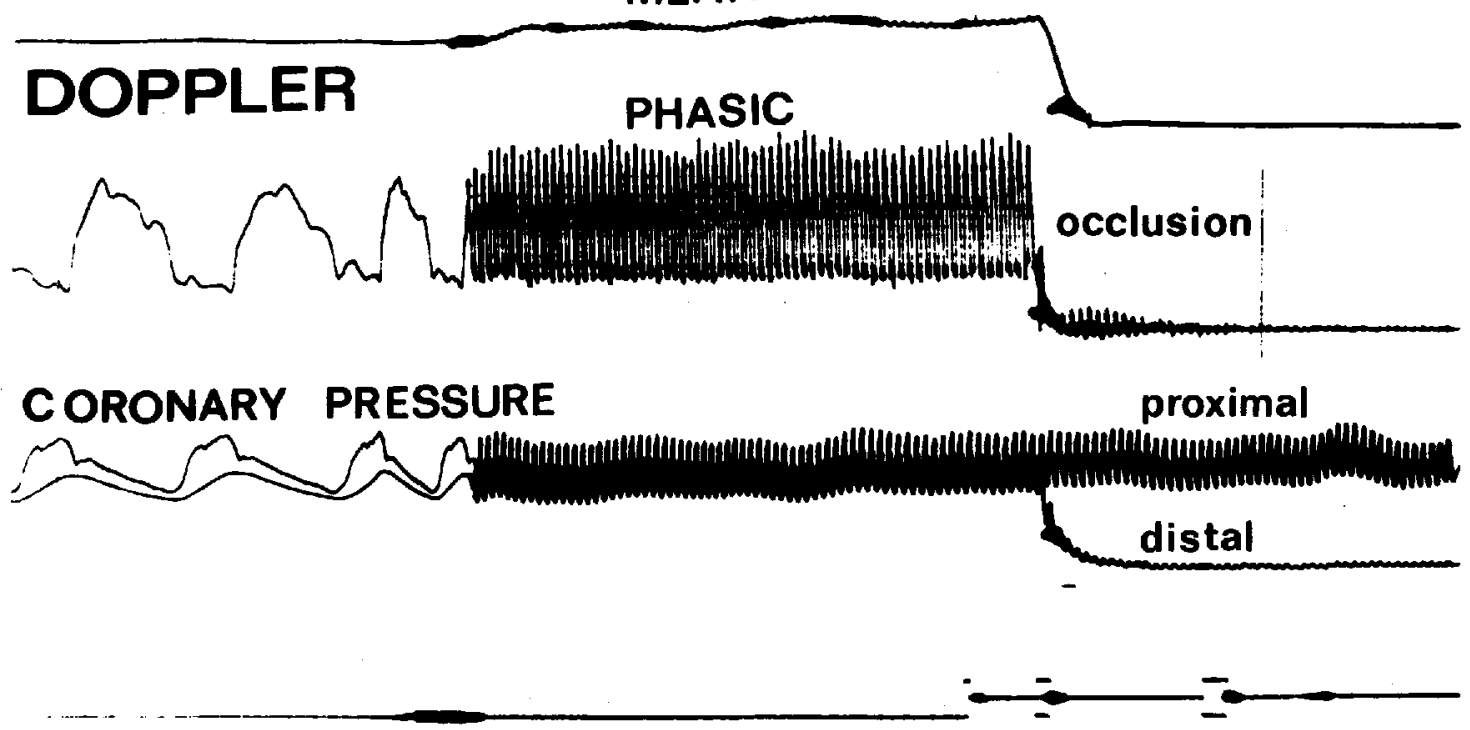

Figure 2A. ECG leads, mean and phasic Doppler signal, translesional pressure-gradient before and during a balloon inflation. The precordial lead $V_{3}$ shows $S T$-segment elevation.

normal, while patient number nine (Table 1) who underwent a LAD dilatation had an akinetic anterior wall. Patient 12 (Table 1) had longstanding arterial hypertension. The mean number of balloon inflations was 4.11 /patient and ranged from 3-6. The primary success rate of the angioplasty procedures was $100 \%$. Three patients had small shallow dissections of the dilated coronary artery segment after the procedure.

Quantitative Analysis of the Coronary Angiogram (Table 2). The minimal luminal cross-sectional area (mean \pm SD) increased from $1.2 \pm 0.8$ to $3.6 \pm 1.1 \mathrm{~mm} .^{2}$ Percentage diameter stenosis (mean \pm SD) decreased from $61 \pm 11 \%$ to 29 $\pm 10 \%$. Percentage area stenosis (mean \pm SD) decreased from 83 to $10 \%$ to $49 \pm 14 \%$. The interpolated reference area (mean $\pm \mathrm{SD})$ at the site of obstruction was $7.1 \pm 1.6 \mathrm{~mm}^{2}$.
Assessment of Coronary Flow Reserve Using Pharmacologically Induced Hyperemia with Digital Subtraction Cineangiography (PH-DSC, Table 2). In ten patients, PH-DSC was measured before and after angioplasty in the myocardium supplied by the dilated coronary artery. PH-DSC (mean $\pm \mathrm{SD}$ ) increased from $1.1 \pm 0.4$ to $2.2 \pm 0.4$ $(\mathrm{P}<0.001)$. In nine patients, $\mathrm{PH}-\mathrm{DSC}$ was also measured in a coronary artery with no angiographically significant coronary artery disease. PH-DSC (mean $\pm \mathrm{SD}$ ) in these vessels was $3.3 \pm 0.7$ which differed significantly $(\mathrm{P}<0.01)$ from the $\mathrm{PH}-\mathrm{DSC}$ of the dilated coronary artery in the same patients $(2.2 \pm 0.4)$.

Assessment of Coronary Flow Reserve, Using Pharmacologically Induced Hyperemia, with the Doppler Probe. (PH-DOP, Table 2, Fig. 2C). In eight patients, PH-DOP was measured before and 

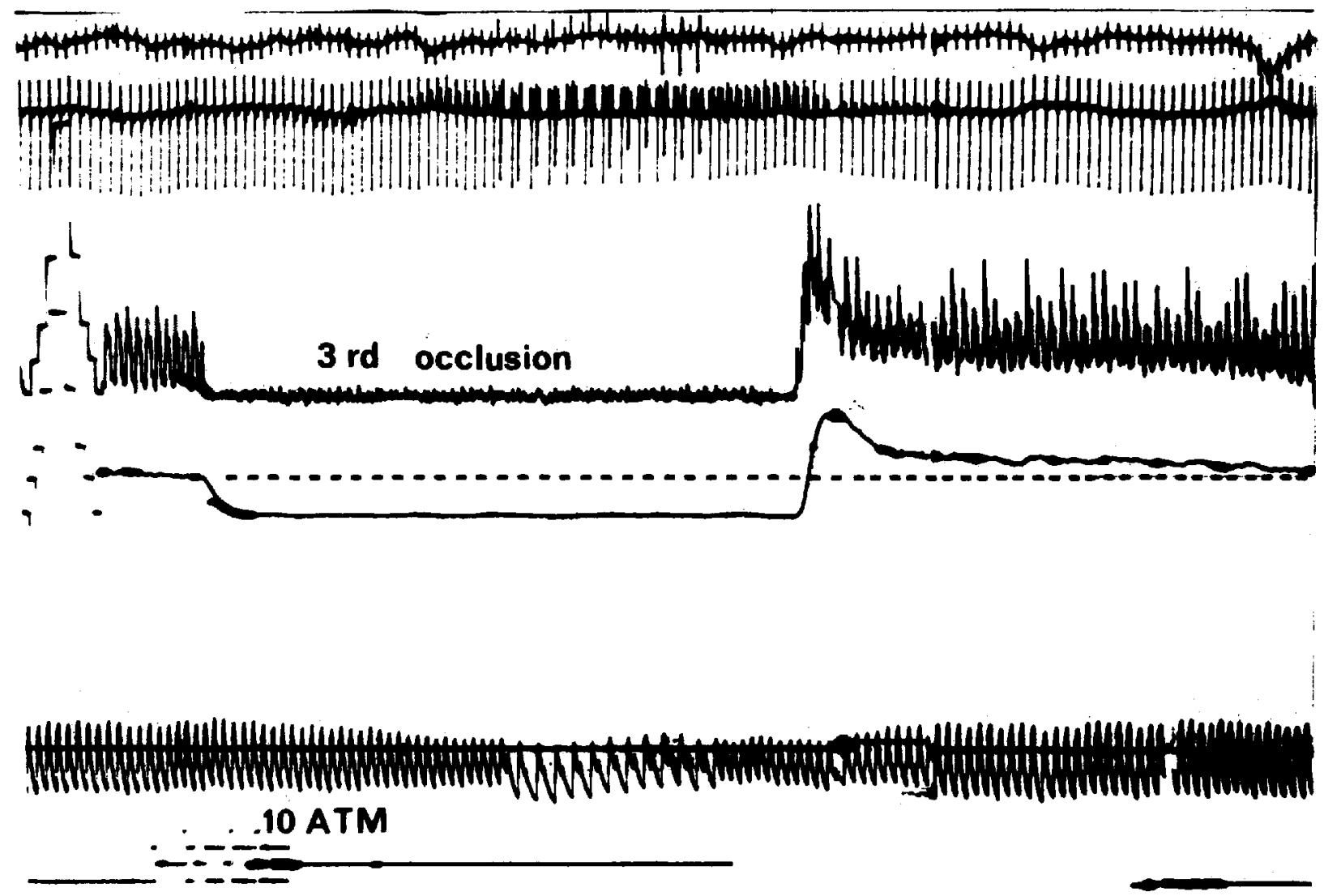

Figure 2B. Example of reactive hyperemia after a third transluminal occlusion using a balloon inflation pressure of 10 atmosphere.

after angioplasty in the dilated coronary artery. The minimal luminal cross-sectional area was 1.5 $\mathrm{mm}^{2}$ before angioplasty and $3.9 \mathrm{~mm}^{2}$ after angioplasty. PH-DOP (mean $\pm \mathrm{SD}$ ) increased from 1.2 \pm 0.3 to $2.4 \pm 0.4(\mathrm{P}<0.001)$. In the remaining five patients no Doppler recording could be made before the angioplasty. They had a mean minimal luminal cross-sectional area before angioplasty of $0.6 \mathrm{~mm},{ }^{2}$ and $3.1 \mathrm{~mm}^{2}$ after angioplasty. Since the guidewire $(0.014$ inch) with a cross-sectional area of $0.12 \mathrm{~mm}^{2}$ had already been passed through the stenosis when these measurements were made, the absence of a recordable Doppler signal indicated an extreme reduction in blood flow velocity in the vessel proximal to the stenotic lesion. The mean PH-DOP after the angioplasty procedure was 2.0 in these five patients, which is comparable to the CFR-DOP of the other eight patients.

Reactive Hyperemia Following the Final BalIoon Inflation (RH, Tables 2 and 3, Fig. 2b). In all
13 patients the postocclusion reactive hyperemia after the final balloon inflation was recorded with the Doppler probe. This RH was of the comparable magnitude as the $\mathrm{PH}$ whether assessed by digital subtraction cineangiography or by the Doppler device (Table 3). Reactive hyperemia and PHDSC were linearly related as shown in Figure 3: $\mathrm{RH}=0.8 \times \mathrm{PH}-\mathrm{DSC}+0.6, \mathrm{r}=0.88, \mathrm{SEE}=0.2$. Reactive hyperemia and PH-DOP were also linearly related as shown in Figure 4: $\mathrm{RH}=0.8$ $\times \mathrm{PH}-\mathrm{DOP}+0.5, \mathrm{r}=0.87, \mathrm{SEE}=0.3$.

Relation Between the Pharmacologically Induced Hyperemia (PH) Measured by Digital Sybtraction Cineangiography (DSC) and by the Doppler Probe (DOP). In seven patients PH-DSC and PH-DOṔ were measured before angioplasty, and in all 13 patients, both measurements were made after the procedure. PH-DSC and PH-DOP were nearly related, see Figure 5: $\mathrm{PH}-\mathrm{DOP}=0.9=\mathrm{PH}-$ $\mathrm{DSC}+0.3, \mathrm{r}=91, \mathrm{SEE}=0.3$. 
1

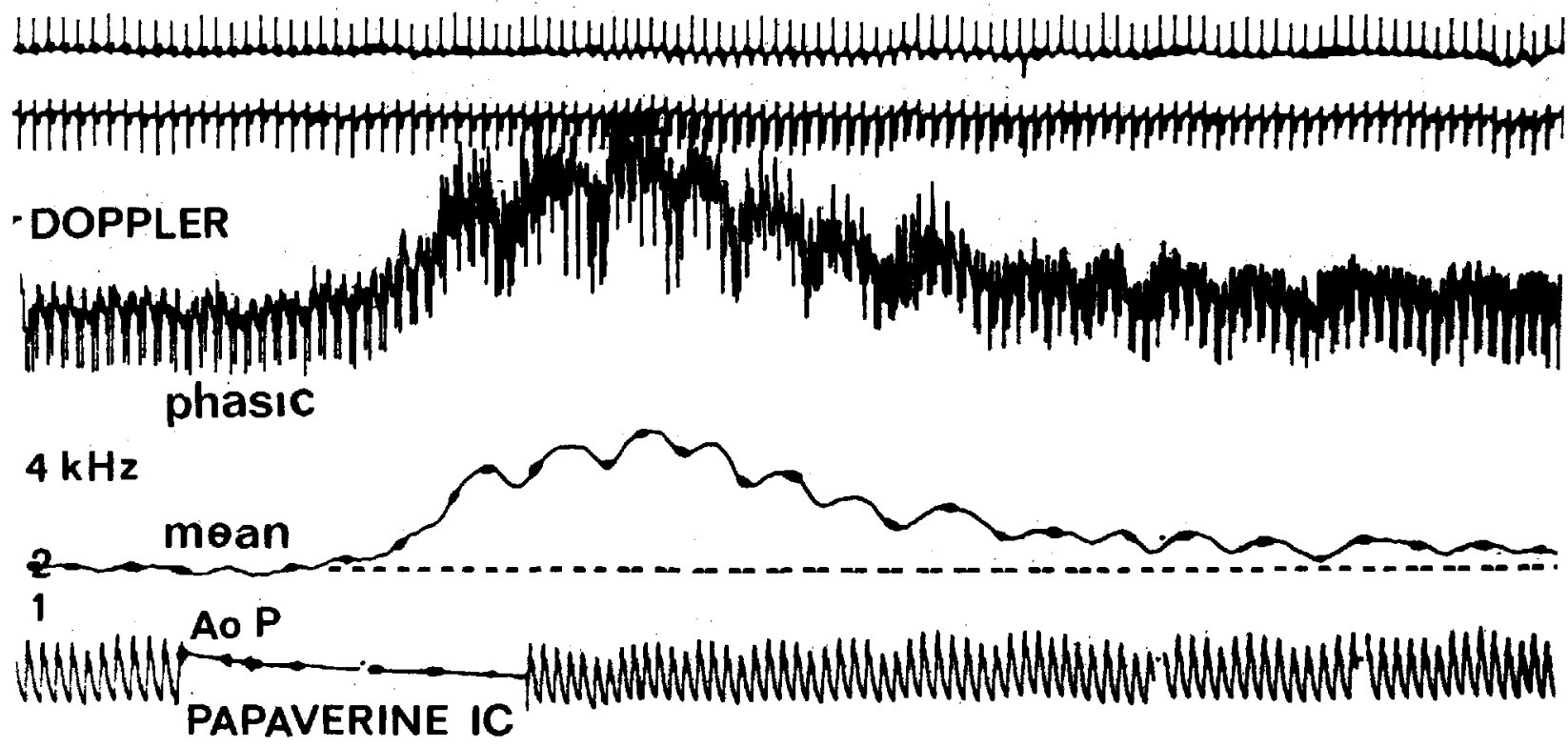

Figure 2C. Example of hyperemia pharmacologically induced with intracoronary (IC) papaverine and measured with the Doppler probe in the prestenotic segment of the dilated vessel. From top to bottom, ECG leads, phasic and mean Doppler signal, and aortic pressure (aoP).

\section{Discussion}

The purpose of the present study was twofold. First, to compare before and after an angioplasty procedure in the same individuals using two different techniques of assessing regional coronary blood flow. Second, to compare the hyperemic response induced by a pharmacological agent (papaverine) with the reactive hyperemia observed following the transient occlusion of a major coronary artery.

Comparison Between the Intracoronary Doppler Blood Flow Velocity Measurements and the Radiographic Determination of Coronary Blood Flow During Hyperemia. Over the last 10 years the relationship between volume and Doppler shift measured from the tip of an intravascular catheter has been extensively validated in peripheral and coronary arteries of anesthetized animals. ${ }^{12,14,18}$ The relationship between the Doppler shift (ranging from
0.5 to $8 \mathrm{kHz}$ ), and the volume flow (ranging from $15 \mathrm{~mL}$ to $400 \mathrm{~mL} / \mathrm{min}$ ) is linear with correlation coefficients generally equal to or greater than $0.95 .^{12,14,18}$ These results have been obtained irrespective of whether the Doppler crystal was end or side mounted on the tip of the catheter. Although excellent correlations between flow velocity and volume flow have been reported in these studies, no attempts have been made to quantify volume flow from the velocity data. Only changes in flow velocity were measured as catheter tip position, crystal angle, and vessel diameter cannot be determined with sufficient accuracy to allow volume flow calculations. Similarly, only relative coronary flow rates can be assessed in myocardial regions of interest by the quantitative digital radiographic determination of time-density curves. Despite the difficulties associated with the use of contrast media and the densitometric rather than geometric assessment of relative vascular volume, a good 


\section{CORONARY FLOW RESERVE DURING ANGIOPLASTY}

Table 1. Clinical Characteristics

\begin{tabular}{|c|c|c|c|c|c|c|c|c|c|c|}
\hline Pat & Age & Sex & NYHA & No of DV & PTCA-vessel & LVEF & Hypertension & Coll circ & Prim success & Dissection \\
\hline 1 & 48 & $\mathbf{F}$ & III & 1 & LAD & 81 & - & - & + & - \\
\hline 2 & 43 & $\mathrm{M}$ & IV & 1 & $\mathrm{LAD}$ & 65 & - & - & + & - \\
\hline 3 & 58 & $\mathbf{M}$ & II & 1 & $\mathrm{RCA}$ & 67 & - & + & + & - \\
\hline 4 & 69 & $\mathbf{M}$ & III & 1 & $\mathrm{RCA}$ & 68 & - & + & + & - \\
\hline 5 & 58 & $\mathbf{M}$ & IV & 2 & LAD & 57 & - & - & + & - \\
\hline 6 & 62 & $\mathbf{M}$ & II & 1 & LAD & 71 & - & - & + & - \\
\hline 7 & 53 & $\mathbf{F}$ & II & 1 & LAD & 70 & - & - & + & + \\
\hline 8 & 56 & $\mathbf{M}$ & III & 1 & LAD & 75 & - & - & + & - \\
\hline 9 & 53 & $\mathrm{M}$ & III & 1 & LAD & 38 & - & - & + & - \\
\hline 10 & 55 & $\mathbf{M}$ & III & 1 & $\mathrm{CX}$ & 63 & - & - & + & + \\
\hline 11 & 53 & $\mathbf{M}$ & III & 1 & $\mathrm{CX}$ & 76 & - & + & + & + \\
\hline 12 & 57 & $\mathbf{F}$ & III & 1 & LAD & 67 & + & - & + & - \\
\hline 13 & 66 & $\mathbf{M}$ & III & 2 & LAD & 68 & - & - & + & - \\
\hline
\end{tabular}

Pat $=$ patient, NYHA $=$ New York Heart Association classification of angina pectoris, No of DV $=$ Number of Diseased Vessels, PTCA = percutaneous transluminal coronary angioplasty, LAD = left anterior descending coronary artery, RCA = right coronary artery, $\mathrm{CX}=$ circumflex coronary artery, $\mathrm{LVEF}=$ left ventricular ejection fraction $(\%)$, Coll circ = angiographic evidence for collateral circulation to the PTCA vessel, Prim success = primary success of PTCA (residual diameter stenosis $<50 \%$ ), Dissection = dissection of

Table 2. Results

\begin{tabular}{|c|c|c|c|c|c|c|c|c|c|c|c|c|c|}
\hline \multirow[b]{3}{*}{ Pat } & \multirow[b]{3}{*}{ Vessel } & \multirow[b]{3}{*}{ No of dil } & \multicolumn{6}{|c|}{ QACA } & \multicolumn{5}{|c|}{ CFR } \\
\hline & & & \multicolumn{3}{|c|}{ Before PTCA } & \multicolumn{3}{|c|}{ After PTCA } & \multicolumn{2}{|c|}{ Before PTCA } & \multicolumn{3}{|c|}{ After PTCA } \\
\hline & & & $\mathrm{OA}$ & DS & AS & $\mathrm{OA}$ & DS & AS & PH-DOP & PH-DSC & RH & PH-DOP & PH-DSC \\
\hline 1 & $\begin{array}{l}\text { LAD } \\
\mathrm{CX}^{*}\end{array}$ & 3 & 1.1 & 64 & 87 & 5.4 & 19 & 35 & - & $\begin{array}{l}1.4 \\
5.0\end{array}$ & 2.2 & 2.1 & 2.0 \\
\hline 2 & LAD & 3 & 2.3 & 54 & 79 & 4.8 & 30 & 51 & 1.0 & - & 2.9 & 2.9 & 3.3 \\
\hline 3 & RCA & 6 & 0.6 & 71 & 91 & 2.9 & 40 & 64 & - & - & 2.8 & 3.0 & 2.8 \\
\hline 4 & $\mathrm{RCA}$ & 3 & 0.3 & 76 & 94 & 2.2 & 41 & 66 & - & - & 1.4 & 1.1 & 1.2 \\
\hline 5 & LAD & 4 & 1.9 & 53 & 78 & 3.3 & 39 & 63 & 0.9 & 0.6 & 2.8 & 2.9 & 2.4 \\
\hline 6 & $\begin{array}{l}\text { LAD } \\
\text { CX }\end{array}$ & 4 & 0.9 & 63 & 86 & 3.5 & 22 & 40 & 0.9 & $\begin{array}{l}1.0 \\
3.0\end{array}$ & 2.0 & 1.8 & 2.0 \\
\hline 7 & $\begin{array}{l}\text { LAD } \\
\text { CX }^{*}\end{array}$ & 4 & 1.6 & 47 & 72 & 3.5 & 30 & 51 & 1.8 & $\begin{array}{l}2.0 \\
2.9\end{array}$ & 2.6 & 2.7 & 2.4 \\
\hline 8 & $\begin{array}{l}\text { LAD } \\
\text { CX* }\end{array}$ & 4 & 0.7 & 65 & 88 & 3.7 & 13 & 24 & 1.1 & $\begin{array}{l}0.8 \\
2.8\end{array}$ & 2.2 & 2.3 & 2.2 \\
\hline 9 & $\begin{array}{l}\text { LAD } \\
C X\end{array}$ & 4 & 1.2 & 59 & 83 & 4.5 & 21 & 37 & 1.3 & $\begin{array}{l}1.1 \\
3.3\end{array}$ & 3.0 & 2.3 & 3.0 \\
\hline 10 & $\begin{array}{l}\mathrm{CX} \\
\mathrm{LAD}^{*}\end{array}$ & 6 & 0.5 & 74 & 93 & 2.5 & 28 & 49 & - & $\begin{array}{l}1.0 \\
3.9\end{array}$ & 2.0 & 1.9 & 1.7 \\
\hline 11 & $\begin{array}{l}\mathrm{CX} \\
\mathrm{LAD}^{*}\end{array}$ & 4 & 0.6 & 69 & 90 & 2.7 & 45 & 69 & - & $\begin{array}{l}0.9 \\
3.1\end{array}$ & 1.7 & 2.0 & 2.2 \\
\hline 12 & $\begin{array}{l}\text { LAD } \\
\text { CX }^{*}\end{array}$ & 5 & 2.9 & 36 & 59 & 5.3 & 23 & 41 & 1.0 & $\begin{array}{l}1.1 \\
2.6\end{array}$ & 2.1 & 2.2 & 1.7 \\
\hline 13 & $\begin{array}{l}\text { LAD } \\
\text { CX }^{*}\end{array}$ & 3 & 0.7 & 58 & 82 & 2.9 & 26 & 44 & 1.2 & $\begin{array}{l}1.4 \\
3.5\end{array}$ & 2.6 & 2.4 & 2.7 \\
\hline
\end{tabular}

Pat = patient, no of dil = number of dilatations, $\mathrm{QACA}=$ quantitative analysis of the coronary angiogram, PTCA = percutaneous transluminal coronary angioplasty, $\mathrm{DS}=$ percentage diameter stenosis, $\mathrm{OA}=$ minimal obstruction area $\left(\mathrm{mm}^{2}\right), \mathrm{AS}=$ percentage area stenosis, CFR = coronary flow reserve, $\mathrm{PH-DOP}=$ ratio of maximal coronary blood flow velocity after intracoronary papaverine as compared to baseline, $\mathrm{PH}-\mathrm{DSC}=$ digital subtraction cineangiographic measurement of CFR, RH = reactive hyperemia after the final dilatation, ${ }^{*}=$ nondiseased coronary artery. 
Table 3. Functional Results of Angioplasty

\begin{tabular}{ll}
\hline & Mean \pm SD \\
\hline PH-DSC & $2.3 \pm 0.6$ \\
PH-DOP & $2.3 \pm 0.5$ \\
RH & $2.3 \pm 0.5$ \\
\hline
\end{tabular}

PH-DSC = pharmacologically induced hyperemia measured with digital subtraction cineangiography, $\mathrm{PH}-\mathrm{DOP}=$ pharmacologically induced hyperemia measured with the Doppler probe and $\mathrm{RH}=$ reactive hyperemia following the final balloon inflation measured with the Doppler probe.

correlation $(r=0.92)$ between electromagnetic flow measurements in the proximal coronary artery and the digital radiographic determinations have been found. ${ }^{24}$ As emphasized by Vogel, the individual ratios must be interpreted bearing in mind the $95 \%$ confidence limits that result in a standard deviation of $0.35 .{ }^{17}$ The two approaches have nothing in common methodologically, and their respective regions of interest (myocardial for the radiographic technique and intravascular for the Doppler technique) are basically different. Since the coronary artery lesions selected in this study were discrete, focal, and not associated with distal narrowing, it is assumed that the flow ratio measured in the myocardial region of interest by the radiographic technique distal to the stenotic lesion reflects the flow velocity ratio measured proximal or distal to the stenotic lesion in the coronary artery. A similar assumption was made by Hodgson et al. in their validation study of the radiographic technique. ${ }^{24}$ Despite these limitations we felt justified in comparing both techniques in

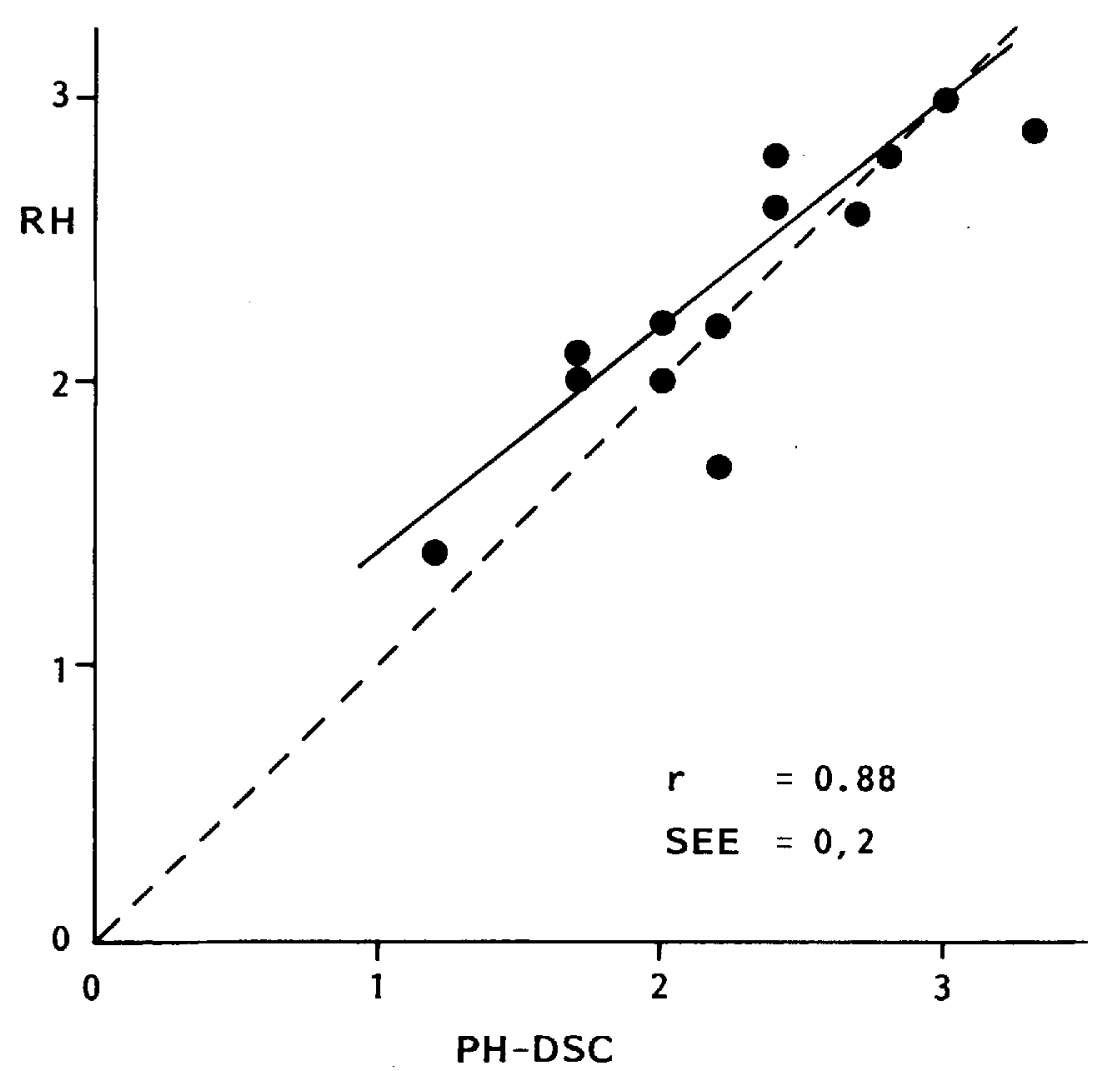

Figure 3. Relationship between reactive hyperemia after transluminal occlusion (RH) assessed by Doppler and pharmacologically induced hyperemia assessed by digital subtraction cineangiography (PH-DSC). The dotted line is the line of identity. The solid line corresponds to the linear regression equation: $\mathrm{RH}=0.8-\mathrm{PH}-\mathrm{DSC}+0.6$ with a regression coefficient ( $r$ ) of 0.88 and a standard error of the estimate (SEE) of 0.2 . 


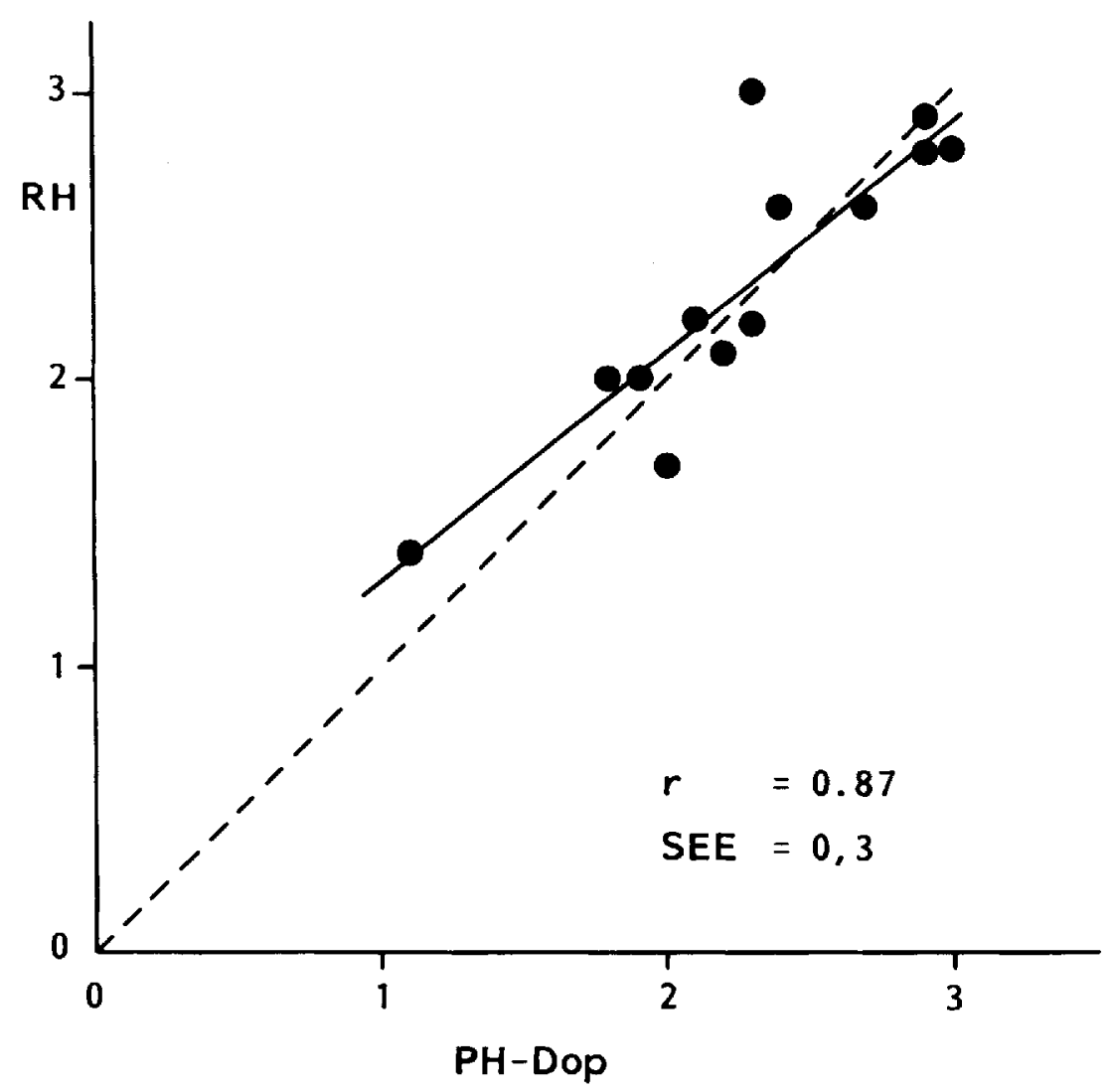

Figure 4. Relationship between reactive hyperemia after transluminal occlusion (RH) assessed by Doppler and pharmacologically induced hyperemia assessed by Doppler. The dotted line is the line of identity. The solid line corresponds to the linear regression equation: RH $=0.8 \times$ PH-Dop +0.5 with a regression coefficient $(r)$ of 0.87 and a standard error of the estimate (SEE) of 0.3 .

the same individuals. A reasonable correlation ( $\mathrm{r}$ $=0.91, \mathrm{SEE}=0.3$ ) between the two methodological approaches was found, suggesting that both techniques are valuable in measuring changes in regional coronary blood flow reserve as a result of an acute intervention.

Comparison Between Pharmacologically Induced Hyperemia and Reactive Hyperemia Following Transient Occlusion. Bookstein and Higgins have shown that in dogs the coronary hyperemic response after an intracoronary injection of adenosine-triphosphate or papaverine is of the same magnitude as the response following a 15 second coronary artery occlusion. ${ }^{25}$ The exact dose of intracoronary papaverine needed to produce maximal coronary arteriolar vasodilation recently has been established. Wilson and White compared the coronary hyperemic response after $4,8,12$ and $16 \mathrm{mg}$ of intracoronary papaverine and reported a maximal hyperemic response after $8 \mathrm{mg}$ in most coronary arteries, and after $12 \mathrm{mg}$ in all coronary arteries. ${ }^{10}$ Papaverine at this dose $(8-12 \mathrm{mg})$ produced a response equal to an intravenous infusion of 0.56 to $0.84 \mathrm{mg} / \mathrm{kg}$ of dipyridamole. The coronary blood flow velocity after papaverine was 4.8 times resting coronary blood flow velocity. The results from this study indicate that in conscious human beings intracoronary papaverine induces a hyperemic response similar to that of transient coronary occlusion, although the reactive hyperemia might have been limited by the presence of the angioplasty catheter. ${ }^{5,26}$ Important in this regard is the size of the angioplasty catheter at the site of the balloon (cross-sectional area: $0.65 \mathrm{~mm}^{2}$ ) 


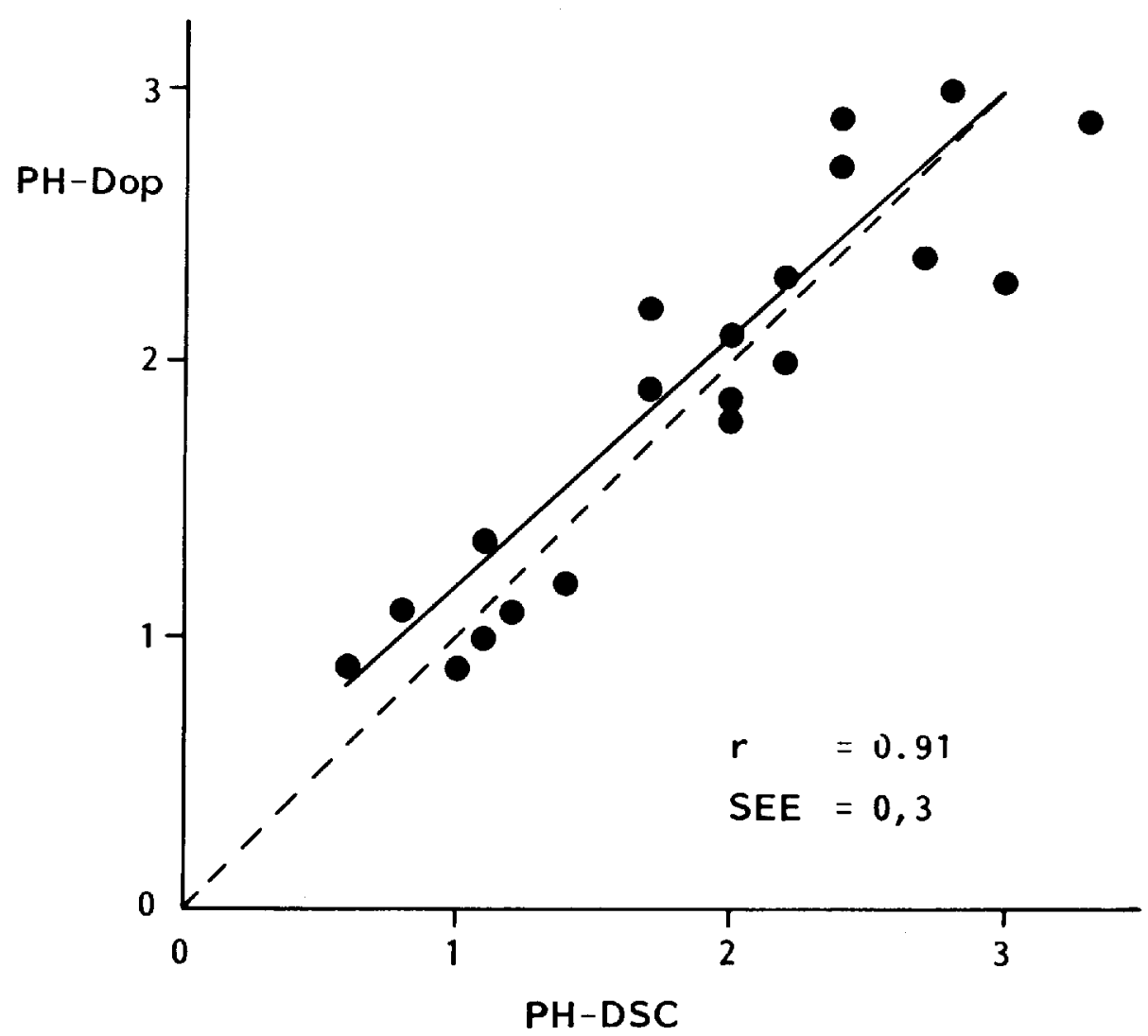

Figure 5. Relationship between pharmacologically induced hyperemia assessed by Doppler (PH-Dop) and pharmacologically induced hyperemia assessed by digital subtraction cineangiography (PH-DSC). The dotted line is the line of identity. The solid line corresponds to the linear regression equation: PH-Dop $=0.9=$ PH-DSC +0.3 with a regression coefficient of 0.91 and a standard error of the estimate (SEE) of 0.3 .

in comparison to the cross-sectional area of the dilated lesion (mean $\pm \mathrm{SD}: 3.6+1.1 \mathrm{~mm}^{2}$ ).

Why is the Coronary Flow Reserve Not Normalized Immediately After the Angioplasty? Although coronary flow reserve in the myocardial region supplied by the dilated vessel increased substantially after the angioplasty procedure, it was not restored to normal. This is in accordance with previous reports in the literature. ${ }^{27,33}$

In our patients, we measured the coronary flow reserve of the adjacent myocardial region supplied by a nonstenotic coronary artery and found a marked difference in response to a vasodilator. This indicates that the abnormal vasodilatory response is restricted to the myocardium supplied by the dilated coronary artery. There are several potential explanations for this phenomenon.

1. Since coronary flow reserve is a ratio between resting flow and maximal coronary blood flow, any increase in resting flow results in a decrease of this ratio. Neither of the techniques we used provided us with absolute measurements of volume flow, and therefore we cannot draw any conclusion regarding the resting coronary volume flow after the angioplasty procedure. However, several authors using the thermodilution technique in the coronary sinus or the great cardiac vein have reported comparable resting volume flows before and after angioplasty. ${ }^{34-38}$ 


\section{CORONARY FLOW RESERVE DURING ANGIOPLASTY}

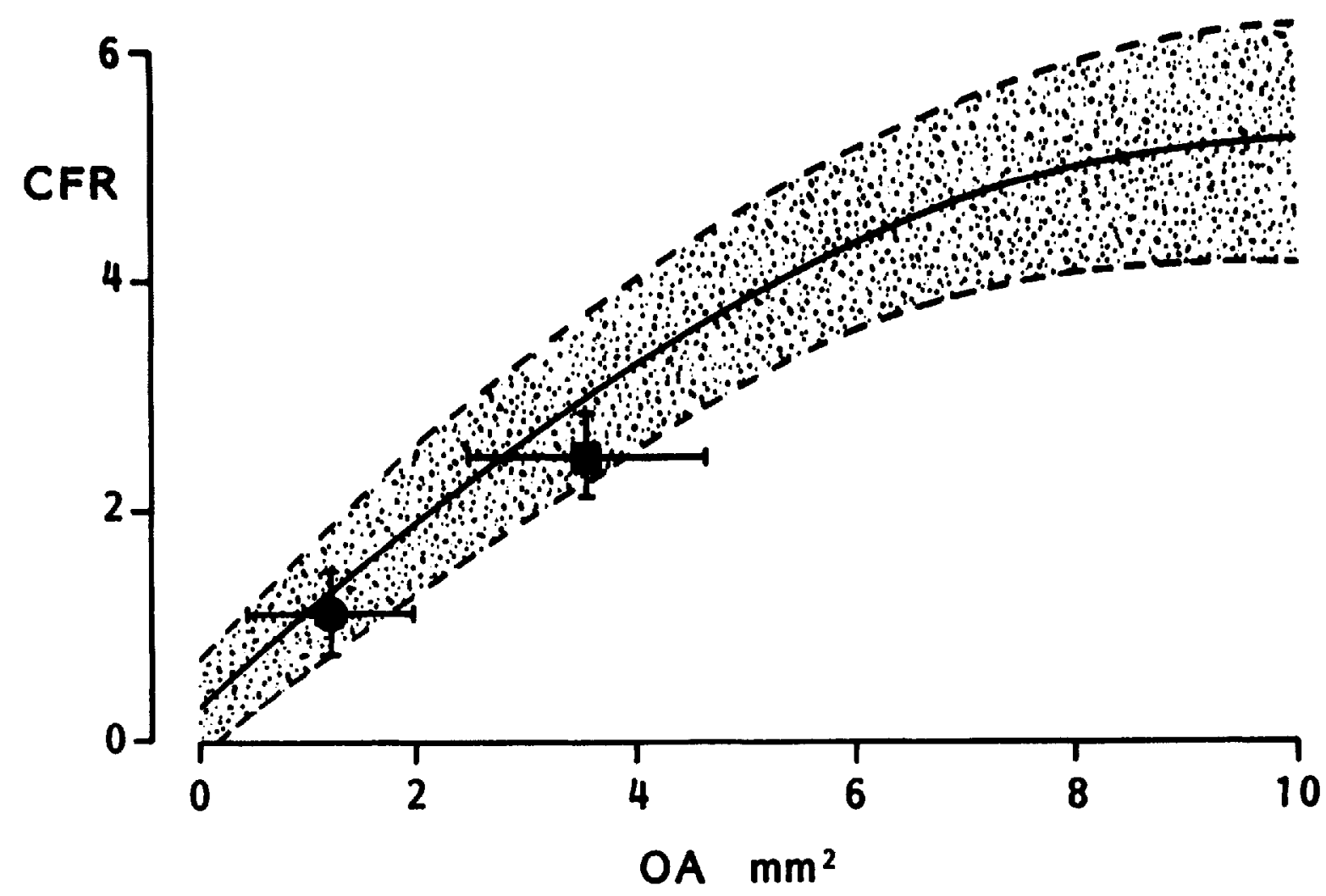

Figure 6. Relationship between coronary flow reserve (CFR) and minimal obstruction area (OA) as previously reported (reference 16). The solid line is the best fit curve, and the shaded area corresponds to the $95 \%$ confidence limits. The mean value and standard deviation of the coronary flow reserve and obstruction area before (circle) and after (square) angioplasty are plotted on this diagram.

2. Metabolic, humoral or myogenic factors could potentially play a role in limiting coronary flow reserve after angioplasty. ${ }^{39}$ The metabolic derangements (lactate, hypoxanthine, potassium) due to the angioplasty seem quickly reversible $^{34,38,40}$ and are therefore not likely to be of major significance in this regard. Although humoral factors such as thromboxane release ${ }^{41}$ may influence vasoactive regulation in a specific subgroup of patients with complicated angioplasty, no scientific evidence has been presented for the persistence of humoral derangements after angioplasty. However, the longstanding reduction in perfusion pressure distal to the stenotic lesion may induce alterations in the complex mechanism of autonomic coronary blood flow autoregulation. ${ }^{15}$ A prolonged period of time might be needed before these abnormalities subside. Finally, Bates et al. postulated that the difference in coronary flow. reserve in men with normal arteries and those who underwent revascularization is related to the atherosclerotic disease process affecting the microvascular reactivity. ${ }^{15}$

3. In contradistinction, the impaired coronary flow reserve could be directly related to the severity of the residual stenosis. Cross-sectional area measured immediately after angioplasty generally increased approximately threefold as a result of the procedure, but remained grossly abnormal and was generally less than half the diameter of the inflated dilating balloon. ${ }^{42}$ In a previous study, our laboratory has established the relationship between cross-sectional area and coronary flow reserve. ${ }^{16} \mathrm{~A}$ measured cross-sectional area of 3.6 $\mathrm{mm}^{2}$ after angioplasty would correspond to an average coronary flow reserve of (Fig. 6): 


$$
\mathrm{CFR}=0.28+0.91 \mathrm{OA}-0.039\left(\mathrm{OA}^{2}\right)=3.0
$$

Therefore, this persisting reduced cross-sectional area is by itself a sufficient explanation for the limited restoration of coronary flow reserve although it does not exclude other contributing pathophysiological mechanisms.

\section{Conclusion}

The data inferred from digital subtraction cineangiography have been corroborated by the subselective intracoronary flow velocity measurements and established this technique as a practical means of assessing regional coronary blood flow reserve in the setting of interventional cardiology. The comparison in human beings between pharmacologically induced hyperemia produced by intracoronary papaverine and the reactive hyperemia after transluminal occlusion confirms previous observations in experimental animals and endorses the use of intracoronary papaverine as a reliable means of inducing maximal coronary vasodilation. Coronary flow reserve is not restored to normal following angioplasty, but its substantial improvement is consistent with the changes in coronary geometry brought about by angioplasty. However, other undefined nonmorphological factors may also play a role in explaining the subnormal coronary flow reserve shortly after dilatation.

Acknowledgments: We are indebted to R.V.D. Perk, J. Ligthart, N. Bruining, R. de Ruiter for their expert technical assistance.

\section{References}

1. Grüntzig AR, Senning A, Siegenthaler WE. Nonoperative dilatation of coronary artery stenosis: percutaneous transluminal angioplasty. N Engl J Med 1979; 301:61.

2. Block PC, Myler RK, Stertzer S, et al. Morphology after transluminal angioplasty in human beings. N Engl J Med 1981; 305:382.

3. Serruys PW, Reiber JHC, Wijns W, et al. Assessment of percutaneous transluminal coronary angioplasty by quantitative coronary angiography: Diameter versus densitometric area measurements. Am J Cardiol 1984; 54I:482.

4. Leimgruber PP, Roubin GS, Hollman J, et al. Restenosis after successful coronary angioplasty in patients with single-vessel disease. Circulation 1986; 73:710.

5. Serruys PW, Wijns W, Reiber JHC, et al. Values and limitations of transstenotic pressure gradients measured during percutaneous coronary angioplasty. Herz 1985; 6:337.

6. Hoffman JIE. Maximal coronary flow and the concept of vascular reserve. Circulation 1984; 70:153.

7. Klocke Fl. Measurements of coronary blood flow and degree of stenosis: current clinical implications and continuing uncertainties. J Am Coll Cardiol 1983; 1:31.

8. Serruys PW, Juilli $\alpha$ ere $\mathrm{Y}, \mathrm{Zijlstra} \mathrm{KJ}$, et al. Coronary Blood Flow velocity during PTCA: a guide-line for assessment of functional results. Am J Cardiol: in press.

9. Zijlstra F, Reiber JC, Juillioere Y, et al. Normalization of coronary flow reserve by percutaneous transluminal coronary angioplasty. Am $J$ Cardiol: in press.

10. Wilson RF, White $\mathrm{CW}$. Intracoronary papaverine: an ideal coronary vasodilator for studies of the coronary circulation in conscious humans. Circulation 1986; 73:444.

11. Zijlstra F, Serruys PW, Hugenholtz PG. Papaverine: the ideal coronary vasodilator for investigating coronary flow reserve: A study of timing, magnitude, reproducibility and safety of the coronary hyperemic response after intracoronary papaverine. Cath Cardiovasc Diagn 1986; 12:298.

12. Cole JS, Hartley CJ. The pulsed doppler coronary artery catheter. Preliminary report of a new technique for measuring rapid changes in coronary artery flow velocity in man. Circulation 1977; 56:18.

13. Wilson RF, Laughlin DE, Ackell PH, et al. Transluminal subselective measurement of coronary artery blood flow velocity and vasodilator reserve in man. Circulation 1985; $72: 82$.

14. Hartley CJ, Cole JS. An ultrasonic pulsed doppler system for measuring blood flow in small vessels. J Appl Physiol $1974 ; 37: 626$.

15. Bates ER, Aueron FM, Le Grand V, et al. Comparative long-term effects of coronary artery bypass graft surgery and percutaneous transluminal coronary angioplasty on regional coronary flow reserve. Circulation $1985 ; 72: 833$.

16. Zijlstra F, van Ommeren J, Reiber JHC, et al. Does quantitative assessment of coronary artery dimensions predict the physiological significance of a coronary stenosis? Circulation 1987; 75:1154.

17. Vogel RA. The radiographic assessment of coronary blood flow parameters. Circulation 1985; 72:460.

18. Sibley DH, Millar HD, Hartley CJ, et al. Subselective measurement of coronary blood flow velocity using a steerable Doppler catheter. JACC 1986; 8:1332.

19. Reiber JHC, Serruys PG, Kooyman Cl, et al. Assessment of short-, medium-, and long-term variations in arterial dimensions from computer-assisted quantification of coronary cineangiograms. Circulation 1985; 71:280.

20. Reiber JHC, Kooijman CJ, Slager CJ, et al. Coronary artery dimensions from cineangiograms; methodology and vasodilation of a computer-assisted analysis procedure. IEEE Trans Med Imaging, 1984; MI-3:131.

21. Reiber JHC, Kooijman CJ, den Boer A, et al. Assessment of dimensions and image quality of coronary contrast catheters from cineangiograms. Cath Cardiovasc Diagn $1985 ; 11: 521$.

22. Sibley DH, Millar HD, Hartley CJ, et al. Subselective measurement of coronary blood flow velocity using a steerable Doppler catheter. JACC 1986; 8:1332.

23. Rentrop KP, Cohen $M$, Blanke $H$, et al. Changes in collateral channel filling immediately after controlled coronary artery occlusion by an angioplasty balloon in human subjects. J Am Coll Cardiol 1985; 5:587. 


\section{CORONARY FLOW RESERVE DURING ANGIOPLASTY}

24. Hodgson JM, LeGrand V, Bates ER, et al. Validation on dogs of a rapid angiographic technique to measure relative coronary blood flow during routine cardiac catheterization. Am J Cardiol 1985; 55:188.

25. Bookstein JJ, Higgins CB. Comparative efficacy of coronary vasodilatory methods. Investigate Radiology 1977 $12: 121$.

26. Leiboff $R$, Bren $G$, Katz $R$, et al. Determinants of transstenotic gradients observed during angioplasty: an experimental model. Am J Cardiol 1983; 52:1311.

27. O'Neill WW, Walton JA, Bates ER, et al. Criteria for successful coronary angioplasty as assessed by alterations in coronary vasodilatory reserve. J Am Coll Cardiol 1984; 3:1382.

28. Wilson RF, Aylward PE, Leimbach WH, et al. Coronary flow reserve late after PTCA.- Do the early alterations persist? (abstract) J Am Coll Cardiol 1986; 7(Suppl):212.

29. Wilson RF, Aylward PE, Talman CL, et al. Does percutaneous transluminal coronary angioplasty restore normal coronary vasodilator reserve? (abstract) Circulation 1985 72(Suppl III):397.

30. Hodgson J McB, Williams DO, Characterization of coronary flow reserve pre- and postsuccessful angioplasty (abstract). Circulation 1985; 72(Suppl III):456.

31. Sibley D, Bulle T, Baxley W, et al. Acute changes in blood flow velocity with successful coronary angioplasty (abstract) Circulation 1976; 74(Suppl. II): 193.

32. Johnson Mm, Wilson RF, Skarton DJ, et al. Coronary lumen area inmediately after angioplasty does not correlate with coronary vasodilator reserve; a video-densitometric study (abstract) Circulation 1986; 74(Suppl II): 193 .

33. Bates ER, Mc Gillem MJ, Beats TF, et al. Angioplasty induced medial injury, but not endothelial denudation. impairs coronary reactive hyperemia (abstract) Circulation 1986; 74(Suppl II):498.
34. Serruys PW, Wijns W, van den Brand $M$, et al. Left ventricular performance, regional blood flow, wall motion, and lactate metabolism during transluminal angioplasty. Circulation 1984; 70:25.

35. Feldman RL, Conti R, Pepine CJ. Regional coronary venous flow responses to transient coronary artery occlusion in human beings. J Am Coll Cardiol 1983; 2:1.

36. Rothman MT, Baim DS, Simpson JB, et al Coronary hemodymamics during percutaneous transluminal coronary angioplasty. Am J Cardiol 1984; 49:1615.

37. Friedman HZ, McGillem MJ, Mancini GBJ, et al. A new method to measure absolute coronary blood flow using standard angioplasty technique. Circulation 1986; 74(Suppl II):497

38. Serruys PW, Piscione F, Wijns W, et al. Myocardial release of hypoxanthine and lactate during percutaneous transluminal coronary angioplasty: a quickly reversible phenomenon, but for how long?". In Serruys PW (Doctoral Thesis, Erasmus University, the Netherlands): Transluminal coronary angioplasty: an investigational tool and a non-operative treatment of acute myocardial ischemia, 1986, p 75 .

39. Marcus KE. The coronary circulation in health and disease. New York, McGraw Hill Book Company, 1983, pp $65,147$.

40. Webb SC, Rickards AF, Poole-Wilson PA. Coronary sinus potassium concentration recorded during coronary angioplasty. Br Heart J 1983; 50:146.

41. Peterson MB, Machay V, Block PC, et al. Thromboxane release during percutaneous transluminal coronary angioplasty. Am Heart J 1986; 111:1.

42. Johnson MR, Brayden GP, Ericksen EE, et al. Changes in cross-sectional area $g$ of the coronary lumen in the six months after angioplasty: a quantitative analysis of the variable response to percutaneous transluminal angioplasty. Circulation 1986; 73:467. 